\title{
BMJ Open Telemedicine for the management of neuropsychiatric symptoms in long- term care facilities: the DETECT study, methods of a cluster randomised controlled trial to assess feasibility
}

\author{
Antoine Piau, ${ }^{1}$ Fati Nourhashemi, ${ }^{1,2}$ Adélaïde De Mauléon, ${ }^{1}$ Achille Tchalla, ${ }^{3}$ \\ Claude Vautier, ${ }^{4}$ Bruno Vellas, ${ }^{1,2}$ Maryline Duboue, ${ }^{1}$ Nadège Costa, ${ }^{5}$ \\ Pierre Rumeau, ${ }^{1}$ Benoit Lepage, ${ }^{6}$ Maria Soto Martin ${ }^{1,2}$
}

To cite: Piau A,

Nourhashemi F, De Mauléon A, et al. Telemedicine for the management of neuropsychiatric symptoms in long-term care facilities: the DETECT study, methods of a cluster randomised controlled trial to assess feasibility. BMJ Open 2018:8:e020982. doi:10.1136/ bmjopen-2017-020982

- Prepublication history and additional material for this paper are available online. To view these files, please visit the journal online (http://dx.doi org/10.1136/bmjopen-2017020982).

Received 7 December 2017 Revised 28 March 2018 Accepted 27 April 2018

Check for updates

${ }^{1}$ Gérontopôle, CHU Toulouse, Toulouse, France

${ }^{2}$ UMR 1027, INSERM, Toulouse, France

${ }^{3} \mathrm{CHU}$ Limoges, Université de Limoges, Limoges, France ${ }^{4}$ Manufacture des Tabacs, Université des Sciences

Sociales-Toulouse 1, Toulouse,

France

${ }^{5}$ Medical Information

Department, CHU Toulouse,

Toulouse, France

${ }^{6}$ Department of Epidemiology, CHU Toulouse, USMR, Toulouse, France

Correspondence to

Dr Antoine Piau;

piau.a@chu-toulouse.fr

\section{ABSTRACT}

Introduction Neuropsychiatric symptoms (NPSs) in elderly patients with dementia are frequent in long-term care facilities (LTCFs) and are associated with adverse events. Telemedicine is an emerging way to provide consultation and care to dependent LTCF residents who may not have easy access to specialty services. Several studies have evaluated telemedicine for dementia care but to date, no study has evaluated its impact in the management of NPS in patients with dementia living in LTCF.

Methods and analysis The Dementia in long-term care facilities: Telemedicine for the management of neuropsychiatric symptoms (DETECT) study is a 24-month multicentre prospective cluster randomised controlled study with two arms: a control arm (usual care) and an intervention arm (telemedicine consultation) for NPSs management. DETECT enrolled 20 LTCFs. The primary outcome is based on the acceptability of the telemedicine among the LTCF staff which will be assessed in the intervention group by quantitative and qualitative indicators. The rate of unscheduled hospitalisations and/ or consultations due to disruptive NPSs, psychotropic drug use and health costs will be described in both groups. Approximately, 200 patients are expected to be recruited. Ethics and dissemination The study protocol was approved and sponsored by the French Ministry of Health. The study received ethical approval from the Toulouse University Hospital Institutional Review Board. We will communicate the final results to the public via conferences and results will also be submitted for publication in international peer-reviewed scientific journals.

Trial registration number NCT02472015.

\section{INTRODUCTION}

Dementia is highly prevalent in long-term care facilities (LTCFs), usually within the range of $50 \%-70 \%$ of residents. ${ }^{1-4}$ Neuropsychiatric symptoms (NPSs) are frequent in elderly patients with dementia affecting $80 \%$ of patients during the disease course $\mathrm{e}^{56}$ :

\section{Strengths and limitations of this study}

- An interventional multicentre prospective randomised controlled trial assessing telemedicine in the management of neuropsychiatric symptom in patients with dementia living in a long-term care facility.

- Special focus on addressing acceptability in real-world setting which is a key issue for future implementation.

- The total duration of follow-up per patient is 2 months, potentially insufficient for analysis of clinical effectiveness.

- The total duration of the study is insufficient to assess organisational aspects and health costs.

hallucinations, delusions, agitation/aggression, dysphoria/depression, anxiety, irritability, disinhibition, euphoria, apathy, aberrant motor behaviour. Among these NPSs, agitation occurs in about 30\%-55\% of patients with dementia and is the most disruptive symptoms to care for. ${ }^{7-11}$ NPSs are associated with a worse disease prognosis, with an increase of formal caregiver burden and inappropriate treatments, and with increased healthcare costs. ${ }^{12-15}$ Some authors emphasise an increased staff turnover in such facilities. ${ }^{16}$ While these symptoms are commonplace, management of NPS is a challenge both to clinicians and to LTCF staff ${ }^{1718}$ due to the lack of current safe and effective drugs in the treatment of such symptoms. As a consequence, consensus guidelines recommend non-pharmacological approaches to be first-line treatments ${ }^{19}$ as they show the best evidence-based results. ${ }^{20}$ NPS generates inappropriate use of health and emergency facilities $^{21}$ which seems to be the current 
easiest way to treat these patients despite the negative impact on the patient's quality of life (QOL), ${ }^{22} 23$ on functional decline ${ }^{2425}$ and on cost for society. ${ }^{26}$ Furthermore, specialised healthcare services are often not adapted to current patients with dementia and disturbing NPS needs; memory consultations and specialised inpatient units face their own limits in NPS care. Environmental causes of NPS are frequently misidentified; thus, evaluation outside the real-life context is biased, partial and inefficient. Additionally, this evaluation requires a change of setting that might worsen the patient's behaviour.

Several studies have evaluated telemedicine (TM) for chronic pathologies with encouraging results ${ }^{27} 28$ and preliminary data suggest its validity for dementia diagnosis. ${ }^{29}{ }^{30}$ However, to date, no interventional study has evaluated the impact of TM in the management of NPS in demented patients living in LTCF. ${ }^{31-34}$ We hypothesised that TM, as a new way of providing care, would be well accepted by LTCF staff and be more effective than usual care in the management of NPS.

The primary aim of the Dementia in long-term care facilities: Telemedicine for the management of neuropsychiatric symptoms (DETECT) study was to assess the acceptability of TM for the management of patients with dementia with disruptive NPS living in LTCF. This paper describes the study protocol.

\section{METHODS AND ANALYSIS}

The DETECT study is a multicentre, open label, cluster randomised controlled trial, comparing an intervention arm (TM) to a control arm (usual care). The unit of randomisation was the LTCF. It is registered on ClinicalTrials. gov since 1 June 2015 (ClinicalTrials.gov: NCT02472015). The protocol version is V.8, 28 June 2017. LTCFs in the intervention group benefit from a TM programme for the management of NPS and the LTCFs in the control group benefit from usual care. Patients with NPS in usual care can be referred to inappropriate care (use of emergency facilities) or to appropriate care (memory consultations and specialised inpatient units), who also face their own limits and important delays. Twenty LTCFs were recruited and randomised in clusters after their consent: 10 LTCFs in the intervention group ('TM group') and 10 in the control group ('usual care group'). The study involved two expert memory centres: one in Toulouse University Hospital (France) (study coordinator) and one in Limoges University Hospital (investigator centre). The coordinating physicians of the participating LTCFs were the associated medical investigators who requested a consultation to the expert centres when in need.

The inclusion and follow-up procedures are illustrated in figure 1.

\section{Participants and enrolment strategy LTCF recruitment}

All LTCFs from the Toulouse Gerontopôle regional research network were eligible for inclusion. The only exclusion criterion was the absence of provided prior consent from the facility and the absence of a TM system. After initial contact by phone and email, the first 20 LTCFs who gave their informed consent were included. We recruited 10 LTCFs in each group. The LTCFs coordinating physicians were responsible for patient recruitment.

\section{Patient recruitment}

All patients living in the enrolled LTCFs suffering from dementia and presenting disruptive NPS were eligible for inclusion. Patients of both sexes were eligible. The detailed inclusion criteria are:

- Patients aged 65 or older, with dementia diagnosed by a specialist or the general practitioner (GP).

- Patients presenting with a disruptive NPS defined as NPS that can be stressful and/or difficult to manage, based on family or professional assessment (eg, wandering, physical aggression), and which requires a specialist consultation or an unscheduled hospitalisation according to LTCF staff.

- Informed and written consent by the patient or the reliable person when appropriate.

- GP agreement.

The exclusion criteria are as follows:

- Patient's life expectancy less than 6 months, according to GP or coordinator physician.

- Non-agreement of study participation by the patient or the reliable person when appropriate.

The inclusion period began in June 2015, with an expected duration of 24 months. To date, inclusions are almost completed. The patient and/or his caregiver can suspend his/her participation to the trial at any time. The first patient was enrolled on 4 June 2015.

\section{Intervention}

The TM intervention consists of synchronous tele-expertise. After randomisation, 10 LTCFs were equipped with TM in the intervention group. The medical and nursing staff was trained in the TM procedure with normalised training addressing regulations, deontology and a special training on the use of a dedicated TM information system. This training was performed by the regional TM Network in each LTCF of the intervention group.

When a patient presents a disruptive NPS, a TM consultation is planned in the following 72 hours. This first consultation is considered as the inclusion visit (inclusion=T0). During this tele-expertise consultation, both the LTCF and Memory Clinic medical and nursing staffs participate. The session is led by a geriatrician trained in NPS management along with the geriatric department nurses. The LTCFs stakeholders are the coordinator physician, nurses, psychologists and patient's GP when possible. The patient's presence may be required based on the physician's assessment. The evaluation and diagnostic process is the first step of any medical care process. When a multidisciplinary consensus is obtained, the team can establish a tailored plan with therapeutic priorities, 


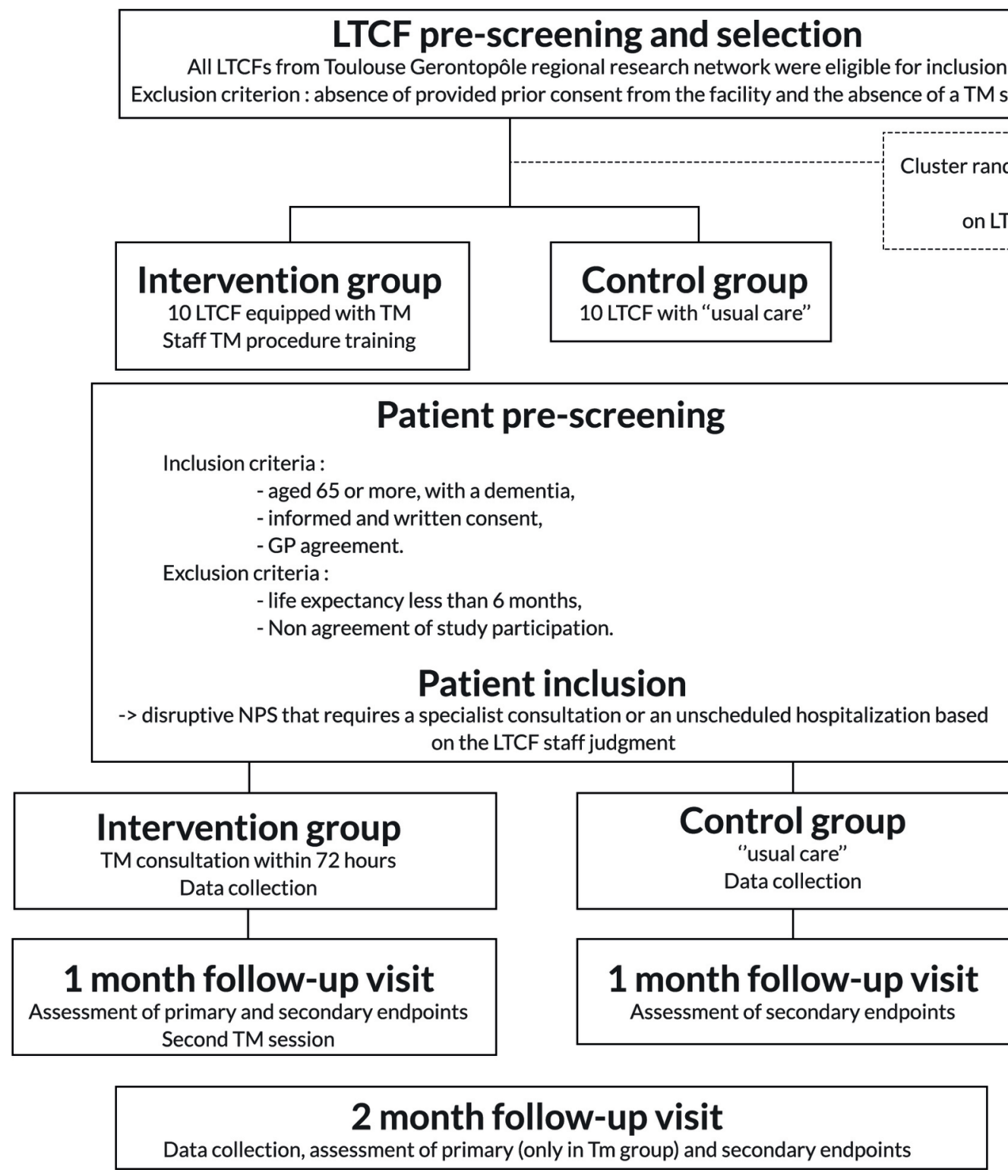

Figure 1 Inclusion and follow-up procedures. GP, general practitioner; LTCF, long-term care facility; NPS, neuropsychiatric symptom; TM, telemedicine.

overall strategy and follow-up plan. A full record is established containing the pharmacological and non-pharmacological strategies proposed for managing NPS and is sent to both, the GPs and the LTCF physician coordinator and staff. For instance, in the case of a patient presenting with aggressive behaviour during the shower, special attention will be given to potential pain or anxiety. Factors related to the professional caregiver (eg, communication skills, non-verbal language) and environmental factors (eg, water temperature or the presence of a mirror) will be analysed. A second follow-up TM consultation takes place at 1 month (T1) in a similar way.

\section{Randomisation}

Randomisation was done with STATA V.11.2. A cluster randomisation was performed with a 1:1 ratio from an anonymised LTCF research network database after their consent (excluding LTCFs that did not provide prior consent or with a number of beds under 60$).{ }^{1}$ The randomisation was stratified on two LTCF characteristics: the region (around Toulouse or around Limoges) and the presence of an Alzheimer unit in the facility. The allocation procedure was conducted by the Department of Epidemiology of the Toulouse University Hospital in Toulouse, France. At enrolment, a fax is sent by the DETECT study group to this department, together with the LTCF code and information regarding assignment.

\section{Outcome measures}

Our primary outcome measure is based on the acceptability of TM among the LTCF staff only in the intervention group. We chose acceptability as a primary outcome because we think that:

- This study evaluates a modification of providing care, an innovation through usage. 
- The highly critical requirement for obtaining profound changes in care practices hinges on the potential for implementing these new practices in specific, real-life settings.

Acceptability of TM among the LTCF staff, and thus its potential for further implementation, will be assessed by qualitative and quantitative indicators in the intervention group during the inclusion and follow-up period:

1. Quantitative indicators (individual level): (a) the proportion of TM solicitation among included patients with disruptive NPS; (b) the time it takes to obtain a TM consultation; (c) the number of staff participants at each TM consultation. This information is systematically collected by the clinical research associate (CRA) two times in a week during the intervention period.

2. Qualitative indicators: collective semistructured interviews are performed by a sociologist to assess staff overall satisfaction and willingness to adopt such solutions in everyday clinical practice. We extract positive attributes and breaks on change to encourage implementation of TM (strengths, weaknesses, opportunities and threats). The follow-up evaluation allows us to assess changes in attitudes. The content of the interviews is analysed by sociological research approach. We made the choice not to use Likert scales or quantitative scores but to call on social sciences methods.

The qualitative evaluation will be performed thanks to the involvement of experts from human and social sciences specialised in social acceptability research (Laboratoire d'études et de recherches sur l'économie, les politiques et les systèmes sociaux, Université Toulouse 1, LEREPS). Measuring acceptability of a procedure has the disadvantage of confronting people with a preconceived project without any opportunity to modify it if it was not scheduled ahead. In agreement with the social experts' recommendations, we involved the participating LTCFs staff in the project definition phase, from the tele-expertise processes to the qualitative evaluation criteria of its acceptability so that they became consensual enough to avoid debate. The proposed method could be called 'usage assisted innovation'. Two meetings are planned or already completed:

- A first meeting at the study kick-off (completed) to refine unmet needs and to define consensual acceptability measurement criteria.

- A second restitution meeting at the end of the study (planned) to perform a collective notation based on the precited criteria, to propose improvements to these criteria and to conclude on the possible adoption of such a solution for everyday care.

In this method, social acceptability is based on the collective work, and the final evaluation is about making sure that all stakeholder concerns have really been taken into account.

The secondary outcome measures will provide the necessary information to design a future nationwide effectiveness and cost-efficacy study. The secondary outcome measures are based on the measure (at the individual level) and comparison of both arms with regard to:

- The rate of hospitalisations and/or consultations due to disruptive NPS.

- Psychotropic drug use, as collected on the basis of the last medical prescription.

- Health costs.

- Patient's QOL.

- The cluster effect ('design effect').

These data are collected in both arms at baseline, 1 month and 2 months, with the exception of the medical and economic data that are collected at baseline and 2 months.

\section{Sample size}

Because it is a feasibility study, a sample size has not been determined in order to demonstrate a statistical difference between both arms. In this cluster design, we can distinguish two levels. On the LTCFs level, 20 facilities are recruited and randomised in clusters, 10 LTCFs in the intervention group ('TM group') and 10 in the control group ('usual care group'). This should be sufficient to observe interfacility variability and obtain information on the outcomes in the two groups, as well as an estimation of the design effect. On the patient level, recent reports show that the facilities in this research network host an average of 80 residents. $^{12} 43 \%$ of residents are suffering from dementia and $82 \%$ of residents with dementia present NPS. ${ }^{6}$ Thus, if one-third of these patients present a disruptive NPS during the inclusion period, it is estimated that around 200 patients could potentially meet the inclusion criteria.

\section{Research procedure}

\section{Inclusions visits}

During a 24-month inclusion period, patients are included in both arms, whenever they present a disruptive NPS that requires a specialist consultation based on the judgement of the LTCF staff. Participation of each patient in both groups lasts 2 months.

In the intervention group, the preinclusion period was performed by the coinvestigator LTCF coordinating physician. The period separating preinclusion and inclusion allowed the LTCF coordinating physician to present the study information to the patient, to his/her family and to his/her GP and to obtain informed consent. Once informed and written consent (see online supplementary file) from the patient (or the legal representative or the reliable person when appropriate) and the GP agreement are obtained, the patient is included. The inclusion visit (T0) is complete when the TM consultation takes place between the LTCF staff and the Memory Center from the University Hospital.

In the 'usual care' control group, the inclusion visit is performed by the CRA who call the LTCFs two times in a week to identify residents presenting a disruptive NPS which, based on the staff's judgement, requires or has already required (between two contacts with the CRA) a 
specialist consultation or an unscheduled hospitalisation. Once the informed and written consent from the patient (or the legal representative or the reliable person when appropriate) and the GP's agreement are obtained (by the coordinator physician), the patient is included. The CRA then proceed to the data collection.

\section{Follow-up visits}

Participants in both groups receive a follow-up visit at 1 month and at 2 months, including the assessment of secondary endpoints. The 1-month follow-up visit (T1) in the intervention group is led by the LTCF coordinator physician and a second TM session is then proposed under the same conditions. In the usual care group, the CRA simply collect data (T1). The third visit at 2 months (T2) involves both groups. In this visit (T2), assessments are performed and data from secondary outcomes are collected by the CRA. The inclusion and follow-up procedures are illustrated in figure 1.

\section{Data collection}

The patients' outcomes are assessed in both groups. Sociodemographic data (age, sex, date of birth), length of stay in the facility, dementia diagnosis, patient parameters such as hospitalisations and/or consultations due to disruptive NPS, psychotropic drugs use, physical restraints use, falls...). Physical disability is based on the Activity of Daily Living scale. ${ }^{35}$ NPS assessment is based on the Neuropsychiatric Inventory-Clinician rating scale. ${ }^{36}$ QOL of patients is based on quality of life in Alzheimer's Disease (QoL-AD).$^{37}$ Health costs are evaluated at baseline (T0) and at 2-month visit (T1) (see economic assessment below). The outcomes in both groups are presented in table 1 . The study is monitored by a clinical research technician, acting for the coordinating investigator and who is responsible for: the logistics and monitoring of the study, regular reports concerning its state of progress, update of the case report forms (eg, request for additional information), collecting and reporting adverse events. This person works in accordance with the standard operating procedures, in cooperation with the clinical research team.

\section{Economic evaluation}

An economic study involving cost comparison will be performed to analyse possible cost differences between patients presenting NPS and receiving TM and those receiving usual care, at 2 months. This evaluation will focus on patients enrolled in the general scheme for employees of the French National Health Insurance (FNHI). Cost analysis will be performed from the FNHI's (paying agent) perspective, and more widely from society's perspective including the family's point of view. Costs taken into account are: direct medical costs including hospitalisation costs, ambulatory care costs and medical treatment costs; direct non-medical costs including transportation costs. Intangible costs, particularly pain, anxiety and lost QOL will not be assessed.
Table 1 Data collection

\section{Inclusion}

Preinclusion TO

Both arms

Informed consent $\quad \checkmark$

Age
Sex
$\begin{aligned} & \text { Length of stay in the } \\ & \text { facility }\end{aligned}$

Dementia diagnosis

$\begin{array}{llll}\text { ADL } & \checkmark & & \checkmark \\ \text { Psychotropic } & \checkmark & \checkmark & \checkmark\end{array}$

medication

\begin{tabular}{lccc} 
Falls & $\checkmark$ & $\checkmark$ & $\checkmark$ \\
\hline Physical restraints & $\checkmark$ & $\checkmark$ & $\checkmark$ \\
Hospitalisations* $^{*}$ & $\checkmark$ & $\checkmark$ & $\checkmark$ \\
Costs (RUD) & $\checkmark$ & & $\checkmark$ \\
QoL-AD & $\checkmark$ & & $\checkmark$
\end{tabular}

Intervention arm

\begin{tabular}{llll} 
NPI-C & $\checkmark$ & $\checkmark$ & $\checkmark$ \\
TM & $\checkmark$ & $\checkmark$ & \\
\hline
\end{tabular}

*Hospitalisations and/or consultations due to disruptive NPS. ADL, activity of daily living; NPI, Neuropsychiatric InventoryClinician; NPS, neuropsychiatric symptom; QoL, quality of life; TM, telemedicine; RUD, Resource Use of Dementia.

Data will be collected from the FNHI regional agency database and hospitals when appropriate. Data related to informal caregiver time will be collected using the Resource Use of Dementia questionnaire. ${ }^{38}$ Hospital stays will be valued from the French diagnosis-related group. Ambulatory and transportation costs (ie, visits, medical and paramedical acts) will be valued from the appropriate reimbursement rate given by the FNHI. Informal care time will be valued using the replacement cost approach. $^{39}$

\section{Statistical analysis}

In a first descriptive stage, we will describe baseline characteristics of the facilities and of the included patients for each group.

The main analysis will be carried out among the included patients in the intervention group. The proportion of TM solicitation for NPS management in the intervention group will be estimated during the 24 months of inclusions with a $95 \%$ CI. This proportion is defined as the proportion of residents who will benefit from a TM consultation for their management among the included residents (residents who present a disruptive NPS). The TM delivery period and the number of effective participants (hospital and LTCF staff) will be described (mean, median, SD, minimum, maximum, quartiles). 
The hierarchical structure of the data will be taken into account in calculating CIs.

For secondary outcomes, the rate of hospitalisations and/or consultation due to disruptive NPS, the prevalence of psychotropic drugs use and health costs will be described over the 2-month follow-up in both groups. Mixed models with a random intercept (corresponding to the cluster units) will be applied to handle within cluster correlation; models will be adjusted for potential confounders. An estimation of the cluster effect ('design effect') will also be performed over the 2-month follow-up in both groups to estimate more accurately the sample size of a nationwide effectiveness demonstration study. Qualitative variables will be described by the percentages of both modalities. Quantitative variables will be expressed by means, $\mathrm{SD}$, minimum, maximum, quartiles and medians. Multiple imputations will be used in order to handle missing data. Statistical analyses will be performed with STATA software. Analyses will be conducted by the Department of Epidemiology of the Toulouse University Hospital in Toulouse, France.

Statistical analysis for costs data will be performed using deterministic and probabilistic sensitivity analyses. In particular, the non-parametric bootstrap method will be used to estimate the $95 \%$ CI of costs. ${ }^{40}$ Statistical cost analysis will be performed with STATA software and conducted by the Unit of Medico-economic Evaluation of the Toulouse University Hospital in Toulouse, France.

\section{Patient and public involvement}

Patients and public were not actively involved in the study design. The results of the study will be disseminated to health professional participants through several meetings and email exchanges.

\section{DISCUSSION}

TM may provide assessment and optimal care to LTCF residents who may not have easy access to specialised services on short notice. TM may provide a more suitable solution than the usual care, as management of NPS requires a comprehensive assessment of the resident in his/her environment in daily life. Moreover, TM could have a positive impact on staff training ${ }^{41-43}$ and even on turnover rates. ${ }^{44}$ In fact, one of the ancillary purposes of this procedure is to allow hospital staff to gain a better understanding of NPS determinants and burdens under real-life conditions. This procedure also helps LTCF staff acquire specialised and up-to-date skills for NPS management: knowledge of the disease, understanding of the symptoms, pharmacological and non-pharmacological treatments, management of crisis and prevention of caregiver exhaustion.

Despite the fact that TM could be an emerging and effective way to provide consultation and care to LTCF residents suffering from dementia, to the authors' knowledge, the DETECT study is the first trial designed to assess the specific impact of a TM programme in the management of NPS in patients suffering from dementia and living in LTCFs.

Our primary aim is to assess the acceptability of the TM solution for the management of demented patients with disruptive NPS in LTCF. Acceptability seems to be a global and relevant primary criterion that meets the overall objectives of this kind of study because real-life implementation of this type of new solution mostly depends on end users' perception. In our opinion, when attempting to support the LTCF staff in managing NPS, DETECT is expected to be an especially acceptable tool and could herald a new organisation of our LTCF care network. We anticipate that TM could provide a more appropriate use of the existing specialised healthcare services. Thus, this could allow us to go beyond current care services in terms of quality and costs, and finally, it could enable skill transfer to LTCF staff. By transmitting knowledge from expert centres to LTCF staff, we could modify their attitude or behaviour and potentially improve the overall care of patients presenting NPS. Thus, by helping LTCF staff to develop coping strategies and to increase their knowledge of the disease, quality of care could be improved and it could promote a more efficient and rational use of care pathways. Moreover, the best evidence-based NPS treatment is demonstrated by non-pharmacological approaches mainly providing education and training to caregivers. ${ }^{20} \mathrm{~A}$ recent meta-analysis reported that staff training decreased antipsychotic prescription among patients with dementia living in LTCFs. ${ }^{45}$

TM may facilitate care of NPS by identifying all underlying causes in real-life conditions and by tailoring a personal and global treatment plan in partnership with the LTCF staff (pharmacological or non-pharmacological treatments including provision of staff education and support, training in problem solving, and targeted therapy directed at the underlying causes for specific behaviours). TM at the service of LTCF staff could be the adequate response for an unbiased and 'ecological' diagnosis, a global evaluation with all staff (more in-depth information) and non-traumatic care.

Concerning the study design, a cluster randomisation for the 20 LTCFs was performed in order to avoid contamination of the intervention group on the control group as a positive consequence of the educational aspect of the team TM intervention on the LTCF staff. The randomisation was stratified on two LTCF characteristics: the region (around Toulouse or around Limoges) and the presence of an Alzheimer unit in the facility to limit possible imbalances between the patients in the two arms as LTCFs could have different operating procedures and patient profiles. Unblinded investigators at each individual LTCF are responsible for enrolling participants on completion of cluster randomisation. Participants may be recruited via a differential selection process. Blinded recruitment is extremely difficult and we think that this is an acceptable bias as the primary aim of this study is to determine acceptability of the TM intervention. Nevertheless, this bias should be addressed more closely in a larger scale 
efficacy study. Thus, even if a potential for unbalanced groups does exist, it will be partially discernible since the baseline characteristics of the facilities and patients enrolled in each group will be described.

The cost analysis evaluation, from the FNHI's perspective, and broadly from society's perspective including the family's point of view, will compare costs between the two arms. This study will provide data on the differences between medical and informal care costs between the TM and usual care arms. Moreover, this study will provide data on the most affected items of expenditure (either positively or negatively) by the TM solution.

The expected patient benefits for this type of intervention are as follows:

- Better and fairer access to specialised care regardless of the LTCF location.

- Unbiased and 'ecological' diagnosis identifying all underlying causes in real-life conditions.

- Global evaluation with all staff (more in-depth information).

- A tailored personal and global treatment plan in partnership with the LTCF staff.

- Non-traumatic care (medical transport and outpatient consultation distress).

- Limitation of psychotropic medication in favour of non-drug therapeutics.

- Improved QOL for patients suffering from dementia and NPS.

For the overall care system, the benefits could be:

- Promotion of knowledge, expertise and skills transfer from expert centres to LTCF staff and vice versa, with a positive spillover effect.

- More efficient and rational use of scheduled consultations and hospitalisations.

- Limitation of inappropriate use of the care pathways, in particular emergency services and medical transport.

- Improvement of the working conditions for LTCF staff.

Finally, results from this study will be used to develop the study design for a subsequent larger national multicentre randomised control trial assessing the efficacy of TM for this indication. Thanks to secondary parameter analysis, this study will allow estimating distribution and expected differences between the two arms (intervention and usual care) and the relevance of judgement criteria and the cluster or design effect.

The final results of this study, such as the acceptability of TM by LTCF staff, might consider whether such an approach needs to be implemented in the national care plan for patients with $\mathrm{AD}$ in the specific context of French LTCFs.

\section{ETHICS AND DISSEMINATION}

Written informed consent is required from all participants (or legal representatives). The trial has been registered in ClinicalTrials.gov: NCT02472015, registered 1 June 2015.
Two times during the study, a clinical research technician acting for the Toulouse University Hospital will conduct an audit of the trial. We plan to communicate the final results to the public via conferences and results will also be submitted for publication in international peer-reviewed scientific journals, approximately 6 months after finishing data collection. All participants will be informed about the final results, if they ask for, as required by French law (article L.1122-1). All authors will have access to the final trial dataset.

Contributors This study protocol was mainly developed by AP, FN and MESM. The first draft of the manuscript was written by AP, MESM and FN. AT, CV, NC and BL contributed to the design of the clinical study. MESM, AT, CV, NC, BL, ADM, BV, MD and PR were involved in the critical revision of the manuscript.

Funding This research is conducted with the support of an interregional PHRC French grant (French Ministry of Public Health, PHRC No 137031 08). This work was sponsored by the University Hospital of Toulouse for regulatory and ethic submission (contact: Aline Meulle meulle.a@chu-toulouse.fr).

Disclaimer Study sponsor has no role in the study design, analysis of data or any other decision related to the study management.

Competing interests None declared.

Patient consent Parental/guardian consent obtained.

Ethics approval The DETECT study received ethical approval from the local institutional review board (№ 2-14-23, 9 September 2014).

Provenance and peer review Not commissioned; externally peer reviewed.

Open access This is an open access article distributed in accordance with the Creative Commons Attribution Non Commercial (CC BY-NC 4.0) license, which permits others to distribute, remix, adapt, build upon this work non-commercially, and license their derivative works on different terms, provided the original work is properly cited and the use is non-commercial. See: http://creativecommons.org/ licenses/by-nc/4.0/

(C) Article author(s) (or their employer(s) unless otherwise stated in the text of the article) 2018. All rights reserved. No commercial use is permitted unless otherwise expressly granted.

\section{REFERENCES}

1. Rolland $Y$, Abellan van Kan G, Hermabessiere S, et al. Descriptive study of nursing home residents from the REHPA network. J Nutr Health Aging 2009;13:679-83.

2. de Souto Barreto P, Lapeyre-Mestre M, Mathieu C, et al. A multicentric individually-tailored controlled trial of education and professional support to nursing home staff: research protocol and baseline data of the IQUARE study. J Nutr Health Aging 2013;17:173-8.

3. Matthews FE, Dening T. Prevalence of dementia in institutional care. Lancet 2002;360:225-6.

4. Helmer C, Pérès K, Letenneur L, et al. Dementia in subjects aged 75 years or over within the PAQUID cohort: prevalence and burden by severity. Dement Geriatr Cogn Disord 2006;22:87-94.

5. Seitz D, Purandare N, Conn D. Prevalence of psychiatric disorders among older adults in long-term care homes: a systematic review. Int Psychogeriatr 2010;22:1025-39.

6. Selbæk G, Engedal K, Bergh S. The prevalence and course of neuropsychiatric symptoms in nursing home patients with dementia: a systematic review. J Am Med Dir Assoc 2013;14:161-9.

7. Brodaty H, Draper B, Low LF. Nursing home staff attitudes towards residents with dementia: strain and satisfaction with work. J Adv Nurs 2003;44:583-90.

8. Bullock R, Hammond G. Realistic expectations: the management of severe Alzheimer disease. Alzheimer Dis Assoc Disord 2003;17(Suppl 3):S80-5.

9. Zwijsen SA, Kabboord A, Eefsting JA, et al. Nurses in distress? An explorative study into the relation between distress and individual neuropsychiatric symptoms of people with dementia in nursing homes. Int J Geriatr Psychiatry 2014;29:384-91.

10. Whall AL, Kim H, Colling KB, et al. Measurement of aggressive behaviors in dementia: comparison of the physical aggression 
subscales of the Cohen-Mansfield agitation inventory and the ryden aggression scale. Res Gerontol Nurs 2013;6:171-7.

11. Lachs MS, Rosen T, Teresi JA, et al. Verbal and physical aggression directed at nursing home staff by residents. J Gen Intern Med 2013;28:660-7.

12. Cohen-Mansfield J, Werner P, Watson V, et al. Agitation among elderly persons at adult day-care centers: the experiences of relatives and staff members. Int Psychogeriatr 1995;7:447-58

13. Schmidt SG, Dichter MN, Palm R, et al. Distress experienced by nurses in response to the challenging behaviour of residents evidence from German nursing homes. J Clin Nurs 2012;21:3134-42.

14. Zuidema SU, de Jonghe JF, Verhey FR, et al. Psychotropic drug prescription in nursing home patients with dementia: influence of environmental correlates and staff distress on physicians' prescription behavior. Int Psychogeriatr 2011;23:1632-9.

15. Pitfield C, Shahriyarmolki K, Livingston G. A systematic review of stress in staff caring for people with dementia living in 24-hour care settings. Int Psychogeriatr 2011;23:4-9.

16. Banaszak-Holl J, Hines MA. Factors associated with nursing home staff turnover. Gerontologist 1996;36:512-7.

17. Normann HK, Asplund K, Norberg A. Attitudes of registered nurses towards patients with severe dementia. J Clin Nurs 1999;8:353-9.

18. de Rooij AH, Luijkx KG, Declercq AG, et al. Professional caregivers' mental health problems and burnout in small-scale and traditional long term care settings for elderly people with dementia in the Netherlands and Belgium. J Am Med Dir Assoc 2012;13:486.e7-486. e11.

19. Gitlin LN, Kales HC, Lyketsos CG. Nonpharmacologic management of behavioral symptoms in dementia. JAMA 2012;308:2020-9.

20. Livingston G, Kelly L, Lewis-Holmes E, et al. A systematic review of the clinical effectiveness and cost-effectiveness of sensory, psychological and behavioural interventions for managing agitation in older adults with dementia. Health Technol Assess 2014;18:1-226.

21. Soto ME, Andrieu S, Villars $\mathrm{H}$, et al. Improving care of older adults with dementia: description of 6299 hospitalizations over 11 years in a special acute care unit. J Am Med Dir Assoc 2012;13:486.e1-486.e6.

22. Wang HE, Shah MN, Allman RM, et al. Emergency department visits by nursing home residents in the United States. J Am Geriatr Soc 2011;59:1864-72.

23. Becker MA, Boaz TL, DeMuth A, et al. Predictors of emergency commitment for nursing home residents: the role of resident and facility characteristics. Int J Geriatr Psychiatry 2012;27:1028-35.

24. Fong TG, Jones RN, Marcantonio ER, et al. Adverse outcomes after hospitalization and delirium in persons with Alzheimer disease. Ann Intern Med 2012;156:848-56.

25. Sampson EL, Gould V, Lee D, et al. Differences in care received by patients with and without dementia who died during acute hospital admission: a retrospective case note study. Age Ageing 2006;35:187-9.

26. Hurd MD, Martorell P, Delavande A, et al. Monetary costs of dementia in the United States. N Engl J Med 2013;368:1326-34.

27. Barlow J, Singh D, Bayer S, et al. A systematic review of the benefits of home telecare for frail elderly people and those with long-term conditions. J Telemed Telecare 2007;13:172-9.

28. van den Berg N, Schumann M, Kraft K, et al. Telemedicine and telecare for older patients-a systematic review. Maturitas 2012;73:94-114.
29. Ramos-Ríos R, Mateos R, Lojo D, et al. Telepsychogeriatrics: a new horizon in the care of mental health problems in the elderly. Int Psychogeriatr 2012;24:1708-24

30. Martin-Khan M, Flicker L, Wootton R, et al. The diagnostic accuracy of telegeriatrics for the diagnosis of dementia via video conferencing. J Am Med Dir Assoc 2012;13:487.e19-487.e24.

31. Edirippulige S, Martin-Khan M, Beattie E, et al. A systematic review of telemedicine services for residents in long term care facilities. $J$ Telemed Telecare 2013;19:127-32.

32. Lyketsos CG, Roques C, Hovanec L, et al. Telemedicine use and the reduction of psychiatric admissions from a long-term care facility. $J$ Geriatr Psychiatry Neurol 2001;14:76-9.

33. Barton C, Morris R, Rothlind J, et al. Video-telemedicine in a memory disorders clinic: evaluation and management of rural elders with cognitive impairment. Telemed J E Health 2011;17:789-93.

34. Azad N, Amos S, Milne K, et al. Telemedicine in a rural memory disorder clinic-remote management of patients with dementia. Can Geriatr J 2012;15:96-100.

35. Katz S, Ford AB, Moskowitz RW, et al. Studies of illness in the aged. The index of adl: a standardized measure of biological and psychosocial function. JAMA 1963;185:914-9.

36. de Medeiros K, Robert P, Gauthier S, et al. The neuropsychiatric inventory-clinician rating scale (npi-c): reliability and validity of a revised assessment of neuropsychiatric symptoms in dementia. Int Psychogeriatr 2010;22:984-94.

37. Logsdon R, Gibbons LE, McCurry SM, et al. Quality of life in Alzheimer's disease: patient and caregiver reports. J Ment Health Aging 1999;5:21-32.

38. Luttenberger K, Graessel E. Recording care time in nursing homes: development and validation of the "RUD-FOCA" (Resource Utilization in Dementia-Formal Care). Int Psychogeriatr 2010;22:1291-300.

39. Costa N, Ferlicoq L, Derumeaux-Burel H, et al. Comparison of informal care time and costs in different age-related dementias: a review. Biomed Res Int 2013;2013:1-15.

40. Briggs A, Gray A. The distribution of health care costs and their statistical analysis for economic evaluation. $J$ Health Serv Res Policy 1998;3:233-45.

41. Spector A, Orrell M, Goyder J. A systematic review of staff training interventions to reduce the behavioural and psychological symptoms of dementia. Ageing Res Rev 2013;12:354-64.

42. Morgan DG, Stewart NJ, D'Arcy C, et al. Work stress and physical assault of nursing aides in rural nursing homes with and without dementia special care units. J Psychiatr Ment Health Nurs 2005;12:347-58.

43. Burgio LD, Stevens A, Burgio KL, et al. Teaching and maintaining behavior management skills in the nursing home. Gerontologist 2002;42:487-96.

44. Elliott KE, Scott JL, Stirling C, et al. Building capacity and resilience in the dementia care workforce: a systematic review of interventions targeting worker and organizational outcomes. Int Psychogeriatr 2012;24:882-94.

45. Richter T, Meyer G, Möhler R, et al. Psychosocial interventions for reducing antipsychotic medication in care home residents. Cochrane Database Syst Rev 2012;12:CD008634. 